\title{
Bohmian mechanics without wave function ontology
}

\author{
Albert Solé \\ Department of Philosophy, Facultat de Filosofia i Lletres, Edifici B, Universitat \\ Autònoma de Barcelona, 08193 Bellaterra (Cerdanyola del Vallès), Barcelona, Spain.
}

E-mail address: Albert.Sole@uab.cat

\begin{abstract}
In this paper I critically assess different three-dimensionalist interpretations of Bohmian mechanics in order to evaluate the prospects of interpreting Bohmian mechanics without committing to a wave function-based ontology. More specifically, my aim is to explore the connection between the debate concerning configuration space realism and another interpretive debate that is specific to Bohmian mechanics. Whereas defenders of the quantum potential approach to the theory claim that Bohmian mechanics is better formulated as quasi-Newtonian, via postulating forces proportional to acceleration, advocates of the guidance approach defend the notion that the theory is essentially first-order and incorporates causal concepts akin to those of Aristotelian physics. Here I analyze whether the desideratum of an interpretation of Bohmian mechanics that is both explanatorily adequate and not committed to the physical existence of the wave function or other entities in configuration space favors one approach to the theory over the other. Contrary to some recent claims in the literature, I argue that the quasi-Newtonian approach based on the idea of a quantum potential does not come at as the winner.
\end{abstract}

Keywords: Bohmian mechanics; quantum mechanics; quantum potential; wave function; explanation; causation. 


\section{Introduction}

2. Wave function ontology?

3. Bohmian mechanics: the guidance vs. the quantum potential approach

4. The DGZ interpretation of Bohmian mechanics

5. Belousek's three-dimensionalist interpretation of Bohmian mechanics

\subsection{Belousek's criticism of the DGZ interpretation}

5.2 Belousek's three-dimensionalist interpretive proposal

6. Suárez's propensity interpretation of Bohmian mechanics

7. Two interpretive alternatives presented

8. Conclusions

Acknowledgements

References

\section{Introduction}

Wave function realism is the view that the wave function refers to and faithfully represents a physical substance that exists "out there" in the world-some sort of wave function stuff. Historically, such a view has not enjoyed much credence. Although Schrödinger first attempted to interpret the wave function as a real vibrating process, the difficulties of such a view very soon became apparent. First, the Gaussian wave packet of a free electron quickly spreads out, thus complicating any explanation of the notorious particle character of the electron. Second, and more importantly, the wave function of an N-particle system is not a function in the ordinary three spatial coordinates, but is defined in configuration space; that is, the $3 \mathrm{~N}$-dimensional space each point of which represents a possible configuration of the $\mathrm{N}$ particles in threedimensional space. Therefore, to think of the wave function as a real vibrating process 
would be to make a commitment to the existence of configuration space and very few were at the time willing to accept such revisionary metaphysics. In addition to these difficulties, the fact that there is only a statistical link between the measured properties of a system and the wave function prompted a grossly instrumentalist interpretation of the latter.

The anti-realist attitude towards the wave function seems, however, to be reversed in the context of Bohmian mechanics. As is well known, the ontology of that theory includes particles that describe precise trajectories. Those trajectories depend on the wave function very much as particles' trajectories depend on gravitational and electromagnetic fields in classical mechanics. Because of this analogy, it has become customary in Bohmian mechanics to interpret the wave function as representing a physical field that guides the particles - despite its multidimensional character. ${ }^{1}$ In this paper, I resist such an interpretation and I assess the prospects of interpreting Bohmian mechanics without making a commitment to the physical existence of the wave function or any other entity that is defined in configuration space. In what follows, I will refer to interpretations that are not committed to the existence of the wave function as threedimensionalist interpretations of the theory.

The issue of how to interpret Bohmian mechanics is a topic that has many ramifications. I restrict my considerations to the putative implications that enforcing the three-dimensionalist desideratum may have upon another important interpretive debate within the Bohmian camp. That debate essentially concerns the form of the fundamental law of motion of Bohmian particles and the explanatory resources that are naturally available to the Bohmian. Defenders of what I will call the quantum potential approach to the theory claim that Bohmian mechanics is essentially a second-order theory with a law of motion for Bohmian particles that is identical to Newton's Second Law, except for the addition of a new quantum force arising from a potential energy term that depends on the wave function. Those who are sympathetic to such an approach consider

\footnotetext{
${ }^{1}$ Examples of Bohmians who are committed to wave function realism are: Bell (1987), Valentini (1992), Holland (1993), and Bohm and Hiley (1993).
} 
that the dynamic and explanatory concepts characteristic of Newtonian mechanics also perform a fundamental explanatory role in Bohmian mechanics. ${ }^{2}$ In contrast, according to defenders of the guidance approach, Bohmian mechanics is essentially a first-order theory and it is the velocity, rather than the acceleration, that plays a privileged role. ${ }^{3,4}$ Some authors defend, in addition, the idea that the guidance approach to the theory incorporates dynamic and explanatory concepts that are somehow akin to those that are characteristic of Aristotelian physics.

Although the three-dimensionalist commitment seems, prima facie, totally irrelevant with regards to the disagreement between defenders of the two approaches to Bohmian mechanics, recent developments in the literature seem to indicate quite the opposite. Dürr et al. (1992; 1997) (hereafter, DGZ) were the first authors to develop a fullyfledged three-dimensionalist interpretation of the theory. In broad terms, those authors claim that the wave function must not be interpreted as a physical object, but should be seen as law-like in nature. In addition, DGZ are explicit in rejecting second-order dynamic concepts and openly support to the guidance view. Their interpretation has not escaped criticism and it is dismissed as explanatorily wanting by Belousek (2003) and Suárez (2007). These latter two authors consider the quantum potential approach to be superior in terms of explanatory power and each of them draws on conceptual resources that are characteristic of that approach to build three-dimensionalist interpretation of Bohmian mechanics that purportedly fares better than the DGZ interpretation in accounting for the phenomena.

In what follows, I argue — in contrast to what Belousek and Suárez maintain — that the desideratum of an interpretation of Bohmian mechanics that is both three-

\footnotetext{
${ }^{2}$ Supporters of the quantum potential approach are, for instance, Bohm (1952), Bohm and Hiley (1993) and Holland (1993).

${ }^{3}$ Supporters of the guidance approach are, for instance, Bell (1987), Valentini $(1992 ; 1997)$ and Dürr et al. $(1992 ; 1997)$.

${ }^{4}$ Many authors refer to what I call the quantum potential approach as the 'causal' view of the theory; whereas the guidance approach is sometimes dubbed the 'minimalist' view of the theory. Since both approaches are open to an interpretation of the movement of Bohmian particles in deterministic causal terms, I think that the use of the term 'causal' to refer to just one of them is misleading. I describe both approaches in some detail in $\S 3$ below, but the locus classicus for a characterization of both views is Baublitz and Shimony (1996). Belousek (2003) also offers a lengthy discussion of this interpretive divide.
} 
dimensionalist and adequate from the point of view of explanation does not provide grounds for favoring the quantum potential approach. I do so, first, by arguing that the DGZ interpretation may not turn out to be so explanatorily wanting, if accounts of explanation other than those vindicated by Belousek and Suárez are considered. Second, I introduce two new three-dimensionalist interpretations of the theory that rely only on resources that are characteristic of the guidance approach and, nevertheless, are at least as explanatory as Belousek's and Suarez's own interpretive proposals according to those authors' favorite accounts of explanation.

The paper is structured as follows. In $\S 2$, I briefly motivate the program of devising a three-dimensionalist interpretation of Bohmian mechanics by reviewing some of the problems of wave function realism. In $\S 3$, I properly introduce Bohmian mechanics and the interpretive divide between the guidance and the quantum potential approaches. I place particular emphasis on Valentini's $(1992 ; 1997)$ attempt to interpret the guidance approach to the theory as underwriting the postulate of Aristotelian forces, since this postulate will be highly relevant to my own interpretive proposals. In $\S 4$, I introduce DGZ's interpretation within the guidance approach, carefully assessing the attempt by those authors to provide a nomological reading of the wave function. In $\S 5$, I evaluate both Belousek's (2003) criticism of the DGZ interpretation and the three-dimensionalist interpretive proposal within the quantum potential approach that he offers in reply. In §6, I discuss Suárez's (2007) three-dimensionalist interpretation of Bohmian mechanics, which is also committed to the quantum potential approach. In $\S 7, \mathrm{I}$ introduce two new three-dimensionalist interpretive proposals that operate within the guidance approach and compare them with Belousek's and Suárez's interpretations of the theory, and I show that the new proposals accrue some explanatory advantages. I end with some brief concluding remarks in $\S 8$.

The specific theme of this paper is the contrast between the guidance and the quantum potential views, and its bearing upon the project of devising a satisfactory three-dimensionalist interpretation of the theory. However, I also hope to offer valuable, up-to-date assessments of many of the extant interpretations on offer, thus providing a 
general survey of how well (or badly) we can interpret Bohmian mechanics without wave function ontology.

\section{Wave function ontology?}

Despite its poor historical record, wave function realism has recently been on the up, due in part to the polemic defense of such a view by Albert (1996) and ever increasing interest in the (Everettian) many-worlds interpretation of quantum mechanics. Albert argues that realism about quantum mechanics in any of its interpretations commits one "to think of wave functions as physical objects in and of themselves" and the conclusion he draws from this is that "the space we live in [...] is configuration-space" (p. 277; emphasis in the original). I do not consider that such revisionary metaphysics is untenable per se; but it does pose (at least) two problems. The first, most obvious challenge for the configuration space realist is that they owe us an account of the manifest three-dimensional character of our perception. Let me refer to this as the "problem of perception". A second, not completely unrelated difficulty is what I call the "problem of lacking invariances". This latter problem has to do with the fact that the dynamical symmetries of non-relativistic quantum mechanics are indeed very suggestive of the three-dimensionality of space, since they include translations and rotations in three independent spatial dimensions-but not $3 \mathrm{~N}$. One can always respond that configuration space is richly structured. However, unless independent reasons are provided for postulating such a structure, the assumption seems to be entirely ad hoc.

Albert's (1996) argument in favor of configuration space realism has two parts: the first deals with realities and the second with appearances. As regards reality, Albert considers that our universe is quantum mechanical with the following Hamiltonian:

(1) $\quad \mathrm{H}=\sum_{i=1}^{3 \mathrm{~N}}\left(p_{i}\right)^{2}+\sum_{\substack{j, k=1 \\ j \neq k}}^{\mathrm{N}} \mathrm{V}_{i j}\left(\left[\left(x_{3 j-2}-x_{3 k-2}\right)^{2}+\left(x_{3 j-1}-x_{3 k-1}\right)^{2}+\left(x_{3 j}-x_{3 k}\right)^{2}\right]^{1 / 2}\right)$

From this, he concludes that our universe is $3 \mathrm{~N}$-dimensional. The idea here is that, regardless of our choice of coordinates in configuration space, the trajectory induced by 
(1) will always include states that are non-separable in those coordinates and, consequently, it will not be possible to express the universal wave function as a product of functions of any proper subset of the coordinates. In short, we cannot properly represent all the dynamical possibilities of a quantum universe with Hamiltonian (1) in a space of less than $3 \mathrm{~N}$ dimensions.

When it comes to appearances, Albert invites us to consider what would happen in a classical universe with the very same Hamiltonian (1). He claims that creatures inhabiting such a universe (if there were any) would perceive it as three-dimensional and as containing $\mathrm{N}$ particles. The only reason Albert gives for such a conclusion is that, in a classical universe, the choice of Hamiltonian (1) provides a notion of inter-particle distance - the 3-dimensional inter-particle distance given by $d_{j k} \equiv\left[\left(x_{3 j-2}-x_{3 k-2}\right)^{2}+\left(x_{3 j-1}-x_{3 k-1}\right)^{2}+\left(x_{3 j}-x_{3 k}\right)^{2}\right]^{\frac{1}{2}}$ - that it is uniquely able to play a meaningful physical role since it "reliably measures the degree to which the particles in question can dynamically affect one another, can alter one another's trajectories" (p. 281). Now, in the final twist to his argument, Albert assumes that-despite living in a quantum-mechanical universe with a Hamiltonian that resembles (1)-if we do not look too closely, the appearances we encounter correspond with those of our classical counterparts. Hence, he concludes that although our universe is really $3 \mathrm{~N}$-dimensional, we perceive it as having only three dimensions.

Albert's argument has many critics and all of its premises can be contended to a certain extent. ${ }^{5}$ But even if we concede that Albert succeeds in providing an account of the three-dimensional character of our illusory experience, he clearly leaves the problem of lacking invariances untouched. ${ }^{6}$

\footnotetext{
${ }^{5}$ For criticism see, for instance, Monton (2002; 2006) or Maudlin (2010).

${ }^{6}$ Lewis (2004) claims that the configuration space we live in is in one sense $3 \mathrm{~N}$-dimensional but in another sense three-dimensional. Let us follow him in distinguishing between these two alleged senses of three-dimensionality by means of a subscript. Configuration space is $3 \mathrm{~N}$-dimensional ${ }_{1}$ because the universal wave function requires $3 \mathrm{~N}$ independent parameters; configuration space is three-dimensional ${ }_{2}$ because the quantum dynamics is invariant upon rotations and translations along only three independent spatial directions. With this argumentative strategy, Lewis is not solving the problem of lacking invariances but merely acknowledging it.
} 
The challenges posed by configuration space realism differ when Bohmian mechanics is considered. Albert himself refers to that theory and adopts a rather unorthodox position with regard to it. According to Albert, when applied to Bohmian mechanics, the realist recipe leads to a commitment to the existence of both the wave function and just one particle - the universal particle-in configuration space. If one sticks to this proposal, both the problem of perception and that of lacking invariances stand. Most Bohmians interpret the theory very differently; as postulating a mixed ontology that includes $\mathrm{N}$ particles in three-dimensional space and the wave function in configuration space. Since it is assumed that material objects are made of Bohmian particles, the space we inhabit and the objects we see are three-dimensional. Hence, both the problem of perception and that of lacking invariances are trivially solved. However, such an ontology poses a problem of a new sort, since as the wave function lives in a different space from that of the particles, it becomes rather complicated to tell a coherent causal story about how the former influences the movement of the latter. In what follows, I refer to this problem as the "problem of communication". 7

Now, the advantages of adopting a purely 3-dimensionalist interpretation of Bohmian mechanics can be easily appreciated. As all physical entities are assumed to inhabit one and the very same space, there is no problem of communication. Furthermore, as that space is just the ordinary 3-dimensional space of our experience, neither the problem of perception nor the problem of invariances arises. I take these notorious benefits of a 3-dimensionalist interpretation of Bohmian mechanics to motivate the ensuing exploration of how far we can take Bohmian mechanics without postulating a wave function ontology.

\footnotetext{
${ }^{7}$ One possible way to address this difficulty would be to think of ordinary 3-dimensional space as an appropriate sub-space projection of $3 \mathrm{~N}$-dimensional configuration space. For a critical assessment of this suggestion, see Belousek (2003, pp. 155ff).
} 


\section{Bohmian mechanics: the guidance vs. the quantum potential approach}

Bohmian mechanics is a theory of the motion of particles that is empirically equivalent to non-relativistic quantum mechanics. As we will see, Bohmian mechanics - just like non-relativistic quantum mechanics - can be interpreted in many different ways, but the four following postulates arguably capture a minimal core that is common to all interpretations.

(BM1) The complete state of a closed N-particle system is specified by the wave function and the positions of the particles. The particles' configuration $Q \equiv\left(\vec{Q}_{1}, \vec{Q}_{2}, \ldots, \vec{Q}_{N}\right) \in \mathbb{R}^{3 N}$, where $\vec{Q}_{k} \equiv\left(Q_{k x}, Q_{k y}, Q_{k z}\right) \in \mathbb{R}^{3}$ is the actual position of the $k$ th particle, defines a point in $3 \mathrm{~N}$-dimensional configuration space that is sometimes referred to as the representative point of the system. ${ }^{8}$ The wave function is defined in configuration space; that is, it has the form $\Psi(q, t)$, with $q \equiv\left(\vec{q}_{1}, \vec{q}_{2}, \ldots, \vec{q}_{N}\right) \in \mathbb{R}^{3 N}$ and $\vec{q}_{k} \equiv\left(q_{k x}, q_{k y}, q_{k z}\right) \in \mathbb{R}^{3}$.

(BM2) The temporal evolution of the wave function is given by the linear Schrödinger equation:

$$
i \hbar \frac{\partial \Psi(q, t)}{\partial t}=\left(\sum_{k=1}^{N} \frac{\hbar^{2}}{2 \mathrm{~m}_{k}} \nabla_{q_{k}}^{2}+\mathrm{V}(q, t)\right) \Psi(q, t)
$$

where $\mathrm{V}(q, t)$ is the classical potential of the system, $\mathrm{m}_{k}$ the mass of the $k$ th particle and $\nabla_{q_{k}}{ }^{2} \equiv \vec{\nabla}_{q_{k}} \cdot \vec{\nabla}_{q_{k}}$, with $\vec{\nabla}_{q_{k}} \equiv\left(\partial / \partial q_{k x}, \partial / \partial q_{k y}, \partial / \partial q_{k z}\right)$. It is worth noticing that in Bohmian mechanics the unitary evolution sanctioned by the Schrödinger equation admits no exceptions.

(BM3) The temporal evolution of the configuration of the particles is given by the guidance equation (in what follows, GE):

$$
\mathrm{m}_{k} \overrightarrow{\mathrm{v}}_{k} \equiv \mathrm{m}_{k} \frac{d \vec{q}_{k}}{d t}=\hbar \operatorname{Im}\left(\frac{\vec{\nabla}_{k} \Psi(q, t)}{\Psi(q, t)}\right)
$$

If the wave function is written in polar form $\Psi(q, t)=\mathrm{R}(q, t) \mathrm{e}^{i \mathrm{~S}(q, t) / \hbar}, \mathrm{GE}$ simplifies to:

$$
\mathrm{m}_{k} \overrightarrow{\mathrm{v}}_{k}=\vec{\nabla}_{k} \mathrm{~S}(q, t)
$$

\footnotetext{
${ }^{8}$ Throughout the paper I use lower case letters to refer to generic variables and upper case letters to refer to actual values of the variables.
} 
(BM4) At some initial instant $t_{0}$, the epistemic probability $\rho$ of configuration $Q$ is given by Born's rule:

(5) $\rho\left(Q, t_{0}\right)=\left|\Psi\left(Q, t_{0}\right)\right|^{2}$

Although Bohmian particles always have determinate positions, according to this last postulate our knowledge of those positions cannot be precise and must conform to the distribution given by (5). The temporal evolution sanctioned by both the Schrödinger equation and GE ensures that, if $\rho$ holds at time $t_{0}$, it will hold thereafter unless a measurement occurs.

Having specified the minimal core of Bohmian mechanics, we are now in a position to consider more carefully the differences between the two approaches to the theory mentioned at the beginning of the paper. Advocates of the guidance approach regard GE as the fundamental law of motion of Bohmian particles and would easily concede that the set of postulates just introduced actually exhaust the content of the theory. In contrast, supporters of the quantum potential approach defend the idea that the fundamental dynamics of Bohmian particles is given by the modified Newton's second law (in what follows, MNSL):

$$
\mathrm{m}_{k} \frac{d^{2} \vec{q}_{k}}{d t^{2}}=\overrightarrow{\mathrm{F}}_{k}^{C l}+\overrightarrow{\mathrm{F}}_{k}^{Q P}
$$

where $\overrightarrow{\mathrm{F}}_{k}^{C l} \equiv-\vec{\nabla}_{q_{k}} \mathrm{~V}(q, t)$ and $\overrightarrow{\mathrm{F}}_{k}^{Q P} \equiv-\vec{\nabla}_{q_{k}} \mathrm{U}(q, t)$, with $\mathrm{U}$ being the so-called quantum potential:

$$
\mathrm{U}(q, t) \equiv-\sum_{k=1}^{N}\left(\frac{\hbar^{2}}{2 \mathrm{~m}_{k}}\right) \frac{\vec{\nabla}_{q_{k}}^{2} \mathrm{R}(q, t)}{\mathrm{R}(q, t)}
$$

According to MNSL, Bohmian particles accelerate because of forces. The only difference with Newton's second law is that-apart from the classical forces arising from the classical potential-there is a new quantum force that depends on the wave function. In contrast, from the standpoint of GE, accelerations are not dynamically relevant and it is the velocity of the particles that is directly given in terms of the wave function. $^{9}$

\footnotetext{
${ }^{9}$ According to Belousek (2003), advocates of the quantum potential approach would take (6) as the fifth postulate of the theory and reinterpret (BM3) along the lines I am about to propose.
} 
In order to properly assess the contrast between the quantum potential and the guidance approach, it is important to understand the relation between GE and MNSL. Given that they are different equations, the reader may suppose that they amount to incompatible attributions of trajectories for the Bohmian particles. That is not, however, the case and the disagreement between the two approaches does not concern the Bohmian trajectories themselves but their interpretation. In fact, assuming that the Schrödinger equation holds, MNSL can be obtained by taking the gradient of GE. ${ }^{10}$ It then follows that the set of solutions of GE is a proper subset of the set of solutions of MNSL. To put it differently, every solution of GE is also a solution of MNSL, but the converse is not true since the latter equation is more general. That MNSL admits more solutions than GE can be seen by considering the form of the equations: since it involves a second-order time derivative of position, MNSL requires two boundary conditions - typically both the initial position and velocity-to fix a solution. Hence, the velocity is contingent from the standpoint of this equation. In contrast, to solve the first-order GE, only the initial positions of the particles can be freely specified and the velocities follow from those together with the wave function.

What matters as regards the consistency of the quantum potential approach is that of all the solutions of MNSL, only those that are also solutions of GE turn out to be compatible with the statistical postulate (BM4) and consequently with the standard quantum-mechanical predictions. Therefore, advocates of the quantum potential approach need to get rid of the surplus content afforded by MNSL and they can naturally do so by restricting the otherwise contingent initial velocities so that they conform to the guidance relation at a given initial time:

$$
\mathrm{v}_{0}\left(Q_{0}, t_{0}\right)=\frac{1}{\mathrm{~m}_{k}} \nabla_{q} \mathrm{~S}\left(Q_{0}, t_{0}\right)
$$

The solution of MNSL picked out the initial conditions $\left(Q_{0}, \mathrm{v}_{0}\left(Q_{0}, t_{0}\right)\right)$ is actually a solution of GE. In other words, the dynamical evolution sanctioned by MNSL ensures that if the guidance relation (8) holds at one arbitrary time $t_{0}$, then it holds at all times.

It is clear, then, that GE plays a role within the quantum potential approach, interpreted not as a fundamental dynamical equation but as a selection rule that restricts the set of admissible solutions of MNSL via constraint (8) on the initial conditions.

\footnotetext{
${ }^{10}$ See Holland (1993: pp. 74 and 279-280).
} 
Now, since GE alone is sufficient to derive the trajectories of Bohmian particles and supporters of the quantum potential approach cannot completely dispense with it, one may reasonably ask what the point is of assuming that the true dynamical equation of the theory is the more complicated, pseudo-Newtonian, MNSL. The answer has to do with explanation, since one of the alleged advantages of the quantum potential approach is that it allows all the explanatory resources characteristic of classical Newtonian mechanics to be imported into Bohmian mechanics. That is, through recourse to MNSL and the quantum potential, the movement of Bohmian particles can naturally be interpreted as being caused by forces proportional to accelerations, energy balances can be used in the relevant explanations, and so on. In short, defenders of the quantum potential approach argue that although the recourse to notions of force and energy may turn out to be superfluous from the standpoint of the empirical content of the theory, it is nevertheless essential from the standpoint of explanation.

It is important to notice, however, that Bohmian phenomena do not conform exactly to a Newtonian mold, since the quantum forces $\overrightarrow{\mathrm{F}}_{k}^{Q P}=-\vec{\nabla}_{q_{k}}(\mathrm{U}(q, t))$ arising from the quantum potential are non-classical. First, they depend on the wave function and are not a pre-assigned function of inter-particle distances; second, as the quantum potential is defined in configuration space, the resulting forces are many-body rather that two-body forces; and, third, the quantum forces $\overrightarrow{\mathrm{F}}_{k}^{Q P}=-\vec{\nabla}_{q_{k}}(\mathrm{U}(q, t))$ need not fall off with interparticle distances.

The quantum potential approach and the guidance approach constitute opposing views of the theory. Each approach provides an interpretive framework that is general enough to accommodate many different interpretations. Hence, it is possible to share the approach to the theory but still differ in many important interpretive respects. Whereas many (but not all) authors regard the quantum potential approach as amounting to a reinterpretation of quantum phenomena in quasi-Newtonian terms, some authors have noted that the guidance approach underwrites a dynamical framework that is somehow reminiscent of Aristotelian physics.

Undoubtedly Valentini $(1992 ; 1997)$ is the author who has pushed the Aristotelian interpretation of Bohmian mechanics furthest, with his defense of the idea that the vectors $\vec{\nabla}_{q_{k}} \mathrm{~S}(q, t)$ that appear on the right-hand side of GE can consistently be understood as Aristotelian forces that are proportional, not to acceleration, but to the 
velocity of the particles. ${ }^{11}$ Thus, Valentini interprets GE as conveying genuinely dynamical content, taking $\vec{\nabla}_{q_{k}} \mathrm{~S}(q, t)$ to represent a real force that, having its origin in the wave function, acts upon the $k$ th particle and is the cause of that particle's deviation from absolute rest.

The very idea of postulating Aristotelian forces in the context of a theory that, after all, is Galilean invariant could seem blatantly contradictory. Valentini (1997) fully appreciates the contradiction, but far from giving up his interpretation, he argues that the alleged Galilean symmetry of Bohmian mechanics is not fundamental but is in fact a fictitious symmetry arising from the unnoticed introduction of fictitious Aristotelian forces. Valentini's argument which leads him to such a conclusion runs as I now explain. $^{12}$

Let me consider, for simplicity, two coordinate reference frames, $\Sigma$ and $\Sigma^{\prime}$, such that the origin of the coordinates of $\Sigma^{\prime}$ is moving away from the origin of the coordinates of $\Sigma$ at a constant velocity $\vec{V} \equiv\left(\alpha_{1}, \alpha_{2}, \alpha_{3}\right)$. The coordinates of the two frames are related by the following transformation:

$$
\left\{\begin{array}{l}
q_{i k}^{\prime}=q_{i k}-\alpha_{i} t \\
t^{\prime}=t
\end{array}\right.
$$

where $q_{i k}^{\prime}\left[q_{i k}\right]$ denotes the $i$ th position coordinate $(i=1,2,3)$ of the $k$ th particle $(k=1, \ldots, \mathrm{N})$ in the system of reference $\Sigma^{\prime}[\Sigma]$. Galilean invariance of the Schrödinger equation requires that the expressions for the wave function in the two frames are related as follows:

$$
\Psi^{\prime}\left(q^{\prime}, t^{\prime}\right)=e^{i f(q, t) / \hbar} \Psi(q, t)
$$

where

$$
f(q, t) \equiv \frac{1}{2} \sum_{k=1}^{N} \mathrm{~m}_{k}\left(\sum_{i=1}^{3} \alpha_{i}^{2}\right) t-\sum_{k=1}^{N} \mathrm{~m}_{k}\left(\sum_{i=1}^{3} \alpha_{i} q_{i k}\right)
$$

\footnotetext{
${ }^{11}$ Aristotle is well known to have claimed that, in cases of enforced motion, the force exerted is proportional to the velocity. It is only in this particular regard that one can talk here about Aristotelian forces. In many other respects, the Aristotelian forces appearing on the right-hand side of GE are completely at odds with the principles of the Aristotelian physics. For instance, while the former act at a distance, Aristotle defended a principle of contact between the moved and the mover. For a characterization of the notion of 'force' in Aristotle, see Jammer (1999: Chapter 3).

${ }^{12}$ For a more detailed account of the argument, see Valentini (1997).
} 
and $\Psi^{\prime}[\Psi]$ is the wave function written in terms of $\Sigma^{\prime}[\Sigma]$ coordinates. Given (10), it is easy to show that the modulus, $\mathrm{R}$, and the phase, $\mathrm{S}$, of the wave function transform in the following way:

$$
\left\{\begin{array}{l}
\mathrm{R}^{\prime}\left(q^{\prime}, t^{\prime}\right)=\mathrm{R}(q, t) \\
\mathrm{S}^{\prime}\left(q^{\prime}, t^{\prime}\right)=\mathrm{S}(q, t)+\frac{1}{2} \sum_{k=1}^{N} \mathrm{~m}_{k}\left(\sum_{i=1}^{3} \alpha_{i}^{2}\right) t-\sum_{k=1}^{N} \mathrm{~m}_{k}\left(\sum_{i=1}^{3} \alpha_{i} q_{i k}\right)
\end{array}\right.
$$

From (12), we obtain:

$$
\vec{\nabla}_{q_{k}}^{\prime} \mathrm{S}^{\prime}=\vec{\nabla}_{q_{k}} \mathrm{~S}-\mathrm{m}_{k} \overrightarrow{\mathrm{V}}
$$

This last relation ensures that GE is Galilean invariant and therefore that Bohmian mechanics is too. However, Valentini considers that the transformation above does not reflect a real symmetry of Bohmian mechanics and interprets the term $-\mathrm{m}_{k} \overrightarrow{\mathrm{V}}$ in (13) as a fictitious Aristotelian force the introduction of which preserves the applicability of GE in the primed reference system, $\Sigma^{\prime}$, provided that the origin of $\Sigma$ is at absolute rest. The situation would be analogous to that in which fictitious forces such as the Coriolis force are introduced in the context of classical Newtonian mechanics in order to be able to apply Newton's laws in non-inertial frames such as those attached to the Earth's surface.

If, more generally, we consider the following transformation,

(14) $\left\{\begin{aligned} q_{i k}^{\prime} & =q_{i k}-\frac{1}{2} \beta_{i} t^{2} \\ t^{\prime} & =t\end{aligned}\right.$

so that the origin of $\Sigma^{\prime}$ has a constant acceleration $\vec{a} \equiv\left(a_{1}, a_{2}, a_{3}\right)$ from the standpoint of $\Sigma$, and we assume that the classical potential $\mathrm{V}$ and classical forces $\overrightarrow{\mathrm{F}}_{k} \equiv-\vec{\nabla}_{k} \mathrm{~V}$ transform as follows:

$$
\begin{aligned}
& \mathrm{V}^{\prime}=\mathrm{V}-\frac{1}{2}\left(\sum_{i=1}^{3} a_{i}^{2}\right) \sum_{k=1}^{N} \mathrm{~m}_{k} t^{2}+\sum_{k=1}^{N} \mathrm{~m}_{k} \sum_{i=1}^{3} a_{i} q_{i k} \\
& -\vec{\nabla}_{k}^{\prime} \mathrm{V}^{\prime}=-\vec{\nabla}_{k} \mathrm{~V}-\mathrm{m}_{k} \vec{a}
\end{aligned}
$$

Then, if Newton's second law holds in the inertial frame $\Sigma$, it also holds in the noninertial frame $\Sigma^{\prime}$. 
The usual view is that the transformation (15) does not reflect a real dynamical symmetry of classical mechanics and that the term $-\mathrm{m}_{k} \vec{a}$ in (16) is a fictitious Newtonian force that makes Newton's second law appear invariant. Valentini emphasizes the similarity between transformations (16) and (13). Since the force $-\mathrm{m}_{k} \vec{a}$ is proportional to the mass of the $k$ th particle, its effect on the particle is independent of the mass. Hence, the forces $\left\{-\mathrm{m}_{k} \vec{a}\right\}$ affect all particles equally, this being a clear mark of their being fictitious. However-Valentini notes - exactly the same can be said of the Aristotelian forces $\left\{-\mathrm{m}_{k} \overrightarrow{\mathrm{V}}\right\}$ in (13).

In the classical case we can easily identify fictitious forces since there is clear agreement on the origin and nature of forces, and consequently a standard for unforced motion. More specifically, given that classical forces quickly fall off with distance and originate in particles, an isolated particle sufficiently far away from other matter provides such standard. That is not the case in Bohmian mechanics, since both the Aristotelian forces on the right-hand side of GE and the Newtonian forces on the righthand side of MNSL do not generally fall off with distance. Valentini exploits this disanalogy to argue that although there is a state of absolute rest in Bohmian mechanics, it cannot be effectively detected because of our inability to distinguish genuine Aristotelian forces from fictitious ones.

It is worth noting that if a view such as Valentini's is to be accepted, the disagreement between the guidance approach and the quantum potential approach to Bohmian mechanics goes furthest than expected. In that case, the difference turns out to encompass not only the explanatory resources and the equations of motion that are taken to be fundamental, but also the dynamical symmetries of the theory and consequently the space-time symmetries naturally associated with them.

Valentini completes his interpretation of Bohmian mechanics by endorsing a very idiosyncratic brand of wave function realism. He makes a commitment to the existence configuration space and considers the phase, S, of the wave function-or the "pilot wave" as he calls it - to represent a real field. The true Aristotelian forces are deemed to have their origin in that field. Now, when Valentini attempts to ascertain its nature, he does not hesitate to affirm that it is a field of Aristotelian formal cause (Valentini 1992, p. 17). This claim sounds rather mysterious and has received much criticism; but here I do not need to assess all the aspects of Valentini's interpretation, since only the 
postulating of Aristotelian forces will be of interest when it comes to characterizing my own alternative 3-dimensionalist interpretation of Bohmian mechanics.

\section{The DGZ interpretation of Bohmian mechanics}

DGZ were the first interpreters of Bohmian mechanics not to treat the wave function as a real field and to offer a detailed alternative account of its nature. According to them, Bohmian mechanics is a theory about particles and their movement in ordinary three-dimensional space. The wave function is not a physical entity but it has to do with the law that governs the motion of particles:

The wave function of the universe is not an element of physical reality. We propose that the wave function belongs to an altogether different category of existence than that of substantive physical entities, and that its existence is nomological rather than material. We propose, in other words, that the wave function is a component of a physical law rather than of the reality described by the law. (Dürr et al. 1997, p. 10)

The ontological picture championed by DGZ really is parsimonious; not only do they refuse to credit the wave function with proper citizenship in the realm of physical things, but they also argue that, of all the dynamic properties of the particles, only the configurations need to be postulated in the ontology in order to obtain a consistent and complete interpretation of Bohmian mechanics. That claim has also been made by Bell and an attempt to justify it may proceed given the privileged role that positions have in Bohmian mechanics. ${ }^{13}$ First, positions are the variables that, together with the wave function, completely determine the state of a Bohmian system and therefore they form the supervenient basis to which the rest of the properties of the particles can be reduced. Second, positions are also privileged epistemically, since it is part and parcel of the Bohmian theory of measurement that measurement outcomes always need to be recorded in terms of positions.

\footnotetext{
13 “[Bohmian] particles are not attributed angular momenta, energies, etc, but only positions as functions of time." (Bell 1990, p. 39; emphasis in the original).
} 
As already mentioned in $\S 1$, DGZ take GE to be fundamental and reject any recourse to the quantum potential and other such second-order concepts, thus unequivocally favoring the guidance approach to the theory:

The quantum potential is neither simple nor natural. [...] Since the dynamics for Bohmian mechanics is completely defined by Schrödinger's equation together with the guiding equation, there is neither need nor room for any further axioms involving the quantum potential! [...] Bohmian mechanics should be regarded as a first-order theory, in which it is the velocity, the rate of change of position, that is fundamental in that it is this quantity that is specified by the theory, directly and simply with the second-order (Newtonian) concepts of acceleration and force, work and energy playing no fundamental role. (Dürr et al. 1997, pp. 25-26)

It is hopeless to ground absolute claims of simplicity and naturalness; hence, if DGZ's claims against the quantum potential approach are read this way, they deserve severe criticism. ${ }^{14}$ However, relative to DGZ's own choice of primitive ontology, their preference for GE looks very reasonable. Since, if only particle positions are part of the ontology, it would be extremely odd to postulate a second-order law such as MNSL as fundamental when there is another candidate law - GE - available that gives the rate of change of the positions directly.

Summing up, the ontological framework postulated by DGZ consists of a set of particles endowed only with positions, $\mathrm{Q}_{k}(\mathrm{t})$, that evolve in 3-dimensional space as sanctioned by GE. The motivation for GE itself is that it is the simplest law of movement that includes the wave function and is Galilean invariant. ${ }^{15}$ No dynamic concepts characteristic of classical mechanics are invoked. Neither is there room for Aristotelian forces in this picture, since the inclusion of such forces would come at the cost of downgrading the Galilean invariance to the status of a fictitious symmetry; a maneuver that would be blatantly at odds with DGZ's derivation of GE. This lack of dynamic concepts does not to worry DGZ, who favor a sort of nomological account of explanation according to which it is the mathematical law-the simpler and more compact, the better-that does the explanatory work:

\footnotetext{
${ }^{14}$ See for instance, Belousek (2003, p. 132).

${ }^{15}$ See Dürr et al. (1992, pp. 852ff) for the derivation. Both Brown et al. (1996) and Belousek (2003) criticize such a derivation.
} 
It hardly seems necessary to remark, however, that physical explanation, even in a realistic framework, need not be in terms of classical physics [...] In the present century fundamental physics has moved sharply away from the search for intuitive explanations in favor of explanations having an air of mathematical simplicity and naturalness, if not inevitability, and this has led to an astonishing amount of progress. (Dürr et al. 1997, p. 27)

If we agree with DGZ that laws play a decisive explanatory role, then, were the wave function a component of the law, we would have to concede that the wave function plays an explanatory role as well. Thus, the claim that the wave function is law-like in nature is crucial to evaluating DGZ's explanatory enterprise. The authors provide a lengthy argument to justify such a claim. I will devote the rest of this section to presenting it, providing some criticism and assessing a nomological interpretation of the wave function that is an alternative to that of DGZ. ${ }^{16}$

Briefly, DGZ consider that the wave function in Bohmian mechanics plays a role that is completely analogous to that of the Hamiltonian in classical Hamiltonian mechanics. To support such claim, they point to three sources of analogy between the Bohmian wave function and the classical Hamiltonian. First, both the Hamiltonian and the wave function are highly dimensional objects. In fact, the classical Hamiltonian is defined in phase space: a space that has twice as many dimensions as configuration space. Since it is regarded, not as a part of the physical state, but as the generator of the evolution of that state, nobody attempts to reify it and there is no complaint about its dimensionality. In a similar vein, were the wave function to be regarded simply as a parameter in the law of motion of Bohmian particles, not as a physical object itself, any complaint about its dimensionality would be unjustified. Second, whereas the wave function can be taken to act upon Bohmian particles as suggested by GE or MNSL, according to Bohmian mechanics, there is no action exerted by particles upon the wave function. In other words, the evolution of the configuration of the particles depends on the wave function; but the evolution of the wave function, given by the Schrödinger equation, is completely independent of the actual path followed by the particles. This state of affairs would be odd if we were to think of the wave function as a physical object among other

\footnotetext{
${ }^{16}$ In what follows, I will summarize arguments that can be found both in Dürr et al. (1997) and in Goldstein and Zanghì (2012).
} 
physical objects; but it is the natural thing to expect if the wave function is just a component in the law of motion of the particles.

DGZ note a third source of analogy and compare not the wave function but its logarithm with the classical Hamiltonian $\left(\mathrm{H}_{\mathrm{class}}\right)$. GE can be written as:

$$
\text { (17) } \frac{d Q}{d t}=\operatorname{Der}(\log \Psi)
$$

so that the temporal evolution of the Bohmian configurations is generated by applying a suitable derivative operator 'Der' to the logarithm of the wave function. The classical Hamiltonian generates the temporal evolution of classical state variables, $\xi$, in an analogous way, since Hamilton's equations of motion have the following form:

$$
\text { (18) } \frac{d \xi}{d t}=\operatorname{Der}\left(\mathrm{H}_{\text {class }}\right)
$$

where again 'Der' stands for a suitable derivation. Down at the statistical level, the distribution postulate (BM4) of Bohmian mechanics reads:

$$
\rho \sim|\exp (a \log \Psi)|
$$

with ' $a$ ' being a constant. (19) clearly resembles the classical statistics given by the Boltzmann distribution:

$$
\rho \sim\left|\exp \left(b \mathrm{H}_{\text {class }}\right)\right|
$$

with ' $b$ ' also being a constant. I do not want to claim that these formal analogies are not revealing, but if they have any significance-for instance indicating that it is the logarithm of the wave function and not the wave function itself that deserves an interpretation analogous to that of the classical Hamiltonian-DGZ do not exploit it any further.

These considerations exhaust the positive analogies between the wave function and the classical Hamiltonian and, indeed, a great deal of common suppositions about wave functions clearly go against a nomological interpretation of them. Most of the difficulties faced by such an interpretation have to do with the fact that wave functions are solutions of the Schrödinger equation. Since that equation is regarded as a fundamental law, it would seem that the wave function cannot be nomological as well, 
for it is weird to think of a law that is somehow governed by another law. In addition, solutions of the Schrödinger equation are contingent upon a choice of initial conditions; and that is completely at odds with the necessary character that we attribute to laws. Finally, as a solution of the Schrödinger equation, the wave function has a non-trivial temporal evolution that certainly does not fit with it being essentially law-like.

DGZ attempt to mitigate some of the difficulties by pointing out that most of our intuitions about wave functions do not directly concern the universal wave function but the wave functions attributed to specific subsystems of interest. From the standpoint of Bohmian mechanics, the latter are the so-called conditional or effective wave functions. According to DGZ, it is only the wave function of the whole universe that deserves to be interpreted nomologically and we hardly have any intuitions regarding such an object: it could turn out to be a stationary function, or perhaps even static, uniquely obeying some constraints and hence resembling the Hamiltonian. What we actually do know is how effective wave functions of systems in the laboratory behave and that they obey their own Schrödinger dynamics. Here, DGZ's argumentative strategy consists of showing that the hypothetical assumption of a universal wave function with the features necessary for it to be interpreted as a law is compatible with having effective wave functions of subsystems that reproduce the expected phenomenology.

Before discussing how that can be the case, I introduce some useful definitions. Let A be a subsystem of the universe including $\mathrm{N}$ particles with position variables $x \equiv\left(x_{1}, \ldots, x_{\mathrm{N}}\right)$. Let $y \equiv\left(y_{1}, \ldots, y_{\mathrm{M}}\right)$ be the position variables of all the particles not belonging to A. These latter particles define A's environment. A's conditional wave function at time $t, \psi_{t}^{\mathrm{A}}$, is defined as follows:

$$
\psi_{t}^{\mathrm{A}}(x) \equiv \Psi_{t}(x, \mathrm{Y}(t))
$$

where $\Psi_{t}$ is the universal wave function at $t$ and $\mathrm{Y}(t)$ the actual configuration at $t$ of the particles in A's environment. ${ }^{17}$ Now suppose that the universal wave function can be decomposed in the form:

$$
\Psi_{t}(x, y)=\phi_{t}(x) \theta_{t}(y)+\Psi_{t}^{\perp}(x, y)
$$

\footnotetext{
${ }^{17}$ Obviously, the trajectories $\mathrm{Y}_{k}(\mathrm{t})$ are obtained by solving GE for the particles in A's environment. It is worth noticing that, according to this equation, a universal wave function that is static but has a spatially inhomogeneous phase generates non-trivial currents for the particles.
} 
Where: (a) $\theta_{t}(y)$ and $\Psi_{t}^{\perp}(x, y)$ are functions with macroscopically disjoint supports; and (b) $\mathrm{Y}(t)$ lies within the support of $\theta_{t}(y)$. If conditions (a) and (b) are met, then $\phi_{t}(x)$ is A's effective wave function at $t$. Notice that the effective wave function of a system does not always exist but, when it does, it is equal to its conditional wave function.

Given (21), it is easy to see that even if the universal wave function is static, the conditional wave function, $\psi_{t}^{\mathrm{A}}$, can be time-dependent, since typically the configurations $\mathrm{Y}(t)$ are. It also follows from definition (21) that the temporal evolution of A's particles is given in terms of A's conditional wave function in the usual Bohmian way:

$$
\mathrm{m}_{k} \frac{d \mathrm{X}_{k}}{d t}=\hbar \operatorname{Im}\left(\frac{\nabla_{k} \psi_{t}^{\mathrm{A}}}{\psi_{t}^{\mathrm{A}}}\right)\left(\mathrm{X}_{1}, \ldots, \mathrm{X}_{\mathrm{N}}\right)
$$

It is not always the case, however, that $\psi_{t}^{\mathrm{A}}$ is a solution of a Schrödinger equation with the same Hamiltonian as A; but it can be shown that if $\psi_{t}^{\mathrm{A}}$ is also A's effective wave function, then it obeys a Schrödinger dynamics of its own. ${ }^{18}$ To prove this result, one must assume that the universal wave function is itself a solution of the Schrödinger equation with the Hamiltonian of the universe, as is strictly required by the postulates of the theory. Now, DGZ go further and provide a particular example of a conditional wave function that obeys its own Schrödinger equation that emerges from a purely static universal wave function. According to DGZ, such a situation should be expected to occur with some generality. ${ }^{19}$

As already mentioned, with this line of argumentation DGZ aim to show that it is possible to preserve the phenomenology of effective wave functions of systems in the laboratory even if the wave function of the universe is a static object with the properties required for it to be interpreted as law-like in nature. Since a static function cannot be a solution of the Schrödinger equation, DGZ attempt to justify that the universal wave function is indeed static by invoking the so-called Wheeler-De Witt equation, ${ }^{20}$ which is the fundamental equation for the wave function of the universe in canonical quantum

\footnotetext{
${ }^{18}$ See Dürr et al. (1992, pp. 860ff) for the definition of the effective wave function of a system and an argument leading to the conclusion that effective wave functions obey the Schrödinger dynamics.

${ }^{19}$ To see the particular example and the argument leading to such a conclusion, see Dürr et al. (1996: Section 13).

${ }^{20}$ See De Witt (1967).
} 
cosmology and whose solutions are static. That equation can be schematically represented as:

$$
\text { (24) } \mathcal{H} \Psi=0
$$

where $\mathcal{H}$ is the Hamiltonian constraint in canonical quantum gravity and it involves no explicit time-dependence. DGZ consider that the universal wave function must be obtained as a solution (ideally, the solution) of an equation similar to (24), interpreted as a sort of generalized Laplace equation that allows us to obtain the central parameter, $\Psi$, of what is regarded as the only genuine law of motion of Bohmian mechanics; namely, GE. Hence, the sort of theory that DGZ are after in order to justify their nomological interpretation of the wave function would include GE as a fundamental postulate together with a recipe to obtain the wave function of the universe given some assumptions about the universe's contents. The Schrödinger equation would no longer be a postulate of such a theory; rather it would be derived as a phenomenological consequence of the fundamental postulates concerning the effective wave functions of subsystems that meet certain conditions.

DGZ are certainly pursuing a valuable research program that may contribute greatly to our understanding of the wave function and shed some new light on why our quantum theories have their present form. However, in a context of enquiry that is essentially restricted to interpreting non-relativistic Bohmian mechanics, DGZ's project seems to be quite unsatisfactory as they are no longer interpreting the theory but actually modifying it. It is therefore useful to assess the prospects of the claim that the wave function is nomological in nature in reference to non-relativistic Bohmian mechanics as it stands.

In my opinion, the best that can be done in this direction is as follows. Let us set aside DGZ's speculations about canonical quantum gravity and take the list of postulates given above in $\S 3$ seriously. Hence, the Schrödinger equation is given as a perfectly legitimate axiom of the theory. Still, this does not imply by any means that we are forced to consider that the wave function is part of the physical state. If the wave function, $\Psi$ (or its phase, S), is to be regarded as a parameter in the law of motion of particles, then postulate (BM3) can no longer be properly understood as expressing the law, but a set of laws parameterized by $\Psi$ (or S). In addition, the Schrödinger equation is then naturally interpreted as an auxiliary postulate which serves to provide the 
specific value of the parameter in GE and thus ultimately allows us to determine the law of motion of Bohmian particles that actually applies to our universe.

Summing up, according to this interpretation, the ontology of non-relativistic Bohmian mechanics includes only particles inhabiting three-dimensional space. If the theory is read abstractly, it does not provide the law of motion of the particles but only a nomological schema to which all candidate laws must conform. This is the content of GE. Knowing some facts about our universe (namely, its Hamiltonian and some boundary conditions) we would be able to know which specific instance of the nomological schema held. The Schrödinger equation is the auxiliary function that, taking the relevant information about the contents of our universe as input, gives as output the parameter that saturates the law-like schema.

Certainly, we have here some really odd features. As given by the Schrödinger equation, the wave function parameter is a complicated function of configuration space and time, and therefore the resulting law of motion for Bohmian particles turns out to be more complex than usual classical laws. ${ }^{21}$ This may cause certain discomfort, but a defender of the view can respond that some price has to be paid for having nonrelativistic Bohmian mechanics without wave function ontology.

\section{Belousek's three-dimensionalist interpretation of Bohmian mechanics}

\subsection{Belousek's criticism of the DGZ interpretation}

Belousek (2003) explores the interpretive latitude available in Bohmian mechanics in order to find an interpretation of the theory that is both ontologically tenable and adequate from the point of view of its explanatory power. He dismisses the DGZ interpretation as explanatorily wanting and proposes an interpretive proposal of his own that apparently fares better in explanatory terms. Belousek structures his discussion around the following three desiderata for a satisfactory interpretation of Bohmian mechanics:

\footnotetext{
${ }^{21}$ Belot (2012) introduces some interesting caveats regarding the interpretation of a law that includes explicit time dependence in its formal expression.
} 
(i) All postulated entities should exist in 3-dimensional physical space; (ii) the quantum state $\Psi$ should, in some way, be interpreted in physical (and not merely statistical) terms; and (iii) the physical interpretation of $\Psi$ should adequately underwrite explanation of quantum phenomena. (Belousek 2003, p. 128)

The first desideratum is the 3-dimensionalist requirement we are already familiar with and it is obviously met by the DGZ interpretation. The second condition demands that the role of the wave function must not be restricted solely to providing probabilistic predictions via Born's rule (5). As the trajectories of Bohmian particles depend in one way or another on the wave function, this constraint is trivially met by any interpretation of the theory. It is the last requirement that the interpretation of the wave function must underwrite an adequate explanation of quantum phenomena that is at issue; and Belousek claims that this condition is not met by the DGZ interpretation. In order to appraise whether a given interpretation of the theory satisfies desideratum (iii), we need to be told what counts as an adequate explanation in Bohmian mechanics. Although Belousek does not refer to any specific account of explanation in the literature, he makes it clear through the discussion of particular examples that he requires physical explanation in causal terms.

One of the examples addressed by Belousek concerns the double slit experiment. When the two slits are open, the wave functions originating at each slit interfere with each other and the resulting Bohmian trajectories of the test particle describe a zigzag path until they reach the photographic plate. If an array of such trajectories with initial positions distributed according to the square modulus of the initial quantum state is plotted, it can readily be seen that most trajectories converge at some regions of the plate, whereas the regions in between them hardly receive the terminal points of any trajectories. Consequently, assuming an ensemble of particles all with the same quantum state, the appearance of the usual quantum-mechanical interference pattern on the plate ensues.

Belousek considers that, from the standpoint of Bohmian mechanics, the trajectories of the particles constitute the basic phenomena that that call for an explanation. In fact, he somehow conflates the Bohmian trajectories with the interference pattern itself, taking the former to be a mere redescription of the latter: 
Take, for example, the double-slit experiment. Certainly, assigning an ensemble of particles all with a certain initial quantum state and initial positions distributed according to the amplitude-squared of that state will yield via the guidance equation a set of trajectories that correctly reproduces (as guaranteed by the continuity equation) the expected quantum interference pattern appearing at the screen or photographic plate. To do so, though, is not yet to explain the interference pattern, but rather to redescribe this state of affairs in terms of mathematically precise space-time trajectories. (Belousek 2003, p. 137; emphasis in the original)

This conflation is a mistake, since the epistemic status of the fringe pattern and that of the Bohmian trajectories could hardly be more different. The fringe pattern constitutes the empirical datum while the Bohmian trajectories are, after all, unobservable theoretical posits. As the observed phenomenon, the fringe pattern is precisely the primary explanandum and the specific form and arrangement of the Bohmian trajectories is a very intuitive explanans thereof. The fact that, given a certain initial distribution, the terminal points of the trajectories aggregate on the plate to form fringes, explains the appearance of the interference pattern after many trials of the experiment very nicely. This is not to say that the trajectories do not themselves require an explanation. It would indeed be very satisfying if we could explain why the trajectories have a zigzag shape when the two slits are open; but that is an ulterior explanatory step that should be disentangled from the other.

When it comes to accounting for the Bohmian trajectories, Belousek considers that a fully-fledged realistic interpretation of the quantum potential approach provides the paradigm of a good explanation. According to such an interpretation, the wave function stands for a real field that exerts forces on the particles as sanctioned by MNSL. Between the slits and the screen there is no classical field but, when the two slits are open, there is a complicated quantum field and the quantum potential function has specific peaks and valleys. Hence, the field gives rise to forces that attract the particles to certain regions of the screen and prevent the particles from reaching others. It can be concluded, then, that in a fully-fledged realistic version of the quantum potential approach, the specific shape of the trajectories of the particles is explained in terms of forces acting upon them and the forces are explained in terms of the quantum field. 
The question is whether advocates of the DGZ interpretation can provide an adequate explanation of the Bohmian trajectories. Since DGZ do not reify the wave function and reject, as alien to Bohmian mechanics, all dynamic concepts imported from classical mechanics, they cannot obviously resort to an explanatory story similar to the one given above. We have seen, however, that DGZ favor a brand of nomological account of explanation and the natural thing to say in line with such an account is that the specific form of the Bohmian trajectories in a particular physical situation is explained in terms of the law (namely, GE, including the wave function as a parameter) and the boundary conditions that apply in that physical situation. Belousek considers that this constitutes at best a formal but not a physical explanation of the trajectories and dismisses this account of explanation as amounting to "a collapse of physics into mathematics and the nullification of the very meaning of physical theory" (Belousek 2003, p. 137). His final verdict as regards the DGZ interpretation is that it blatantly fails to meet desideratum (iii).

If desideratum (iii) is understood together with Belousek's strictures concerning explanation in Bohmian mechanics-namely, that the interesting explananda are the trajectories of particles and that the explanantia must be physical causes of them that are somehow related to the wave function - then it should be conceded that his verdict with respect to the DGZ interpretation stands. However, Belousek's two demands are not necessary requirements for adequate explanation in Bohmian mechanics. First, as I argue above, merely postulating Bohmian trajectories together with certain assumptions about the distribution of the initial positions explains, at least in the sense of providing understanding, the statistical phenomenon that forms the empirical basis of both Bohmian mechanics and quantum theory. Hence, even if the Bohmian trajectories were left unexplained, Bohmian mechanics would be more explanatory than ordinary quantum mechanics. Second, to limit explanation to the ascertaining of physical causes leaves out putative explanatory instances that cannot be so easily dismissed. As DGZ themselves notice, a powerful heuristic tool in contemporary physics has to do with the quest for symmetries and it is quite generally agreed that mathematical and geometric explanations play a role in science. The covering law model of explanation has received severe criticism in the literature, mainly because there are many places where explanations are going on but with no proper laws supporting those explanations, and also because of problems when dealing with statistical explananda. Neither of those 
objections applies to the Bohmian case in which we have a deterministic theory with a fundamental law able to underwrite explanation. So it seems that the covering law model of explanation still has a chance in Bohmian mechanics and DGZ may not be that wrong in championing it.

Still, the notion of explanation required by Belousek has an undeniable appeal and it is worth exploring how far we can go in Bohmian mechanics if we are willing to comply with it; that is, if we want to give an account of particle motion in terms of a physical cause over and above the particles themselves. We have seen that a fullyfledged realistic view of the quantum potential approach provides an account along such lines, since the quantum field can be considered to be the physical cause of the particles' motion. However, as the field is defined in configuration space, such an interpretation clearly violates our three-dimensionalist requirement and Belousek's desideratum (i). Belousek attempts to entertain an interpretation of Bohmian mechanics that is able to keep the explanatory power of this fully-fledged realistic view but without postulating the existence of physical entities inhabiting configuration space. Next, I introduce Belousek's interpretation and make some critic remarks.

\subsection{Belousek's three-dimensionalist interpretive proposal}

Since the wave function $\Psi=\operatorname{Re}^{i S / \hbar}$ is a complex-valued field, Belousek considers that it can be attributed no direct physical meaning. The functions amenable to a physical interpretation are, instead, the real-valued $\mathrm{R}$ and $\mathrm{S}$ - the modulus and the phase of the wave function, respectively. Therefore, he attempts to provide an interpretation of both $\mathrm{R}$ and $\mathrm{S}$ that is capable of meeting the desiderata of a tenable three-dimensionalist ontology and a satisfactory explanation.

The physical interpretation of both $\mathrm{R}$ and $\mathrm{S}$ poses no mystery. According to GE, the gradient of $\mathrm{S}$ defines a velocity field in configuration space; whereas according to MNSL, $\mathrm{R}$ is involved in defining a force field in the very same space. Belousek does not interpret such multidimensional fields as physically real entities but considers configuration space to be "an abstract space representing possible histories and interactions in physical space of actually existing particles, the realization of such possibilities being contingent upon actual initial conditions" (p. 162; emphasis in the original). Hence, at each time, only one point of configuration space stands for the 
actual content of a Bohmian universe, the rest of the points represent mere possibilia. According to Belousek, these actual contents include the Bohmian particles inhabiting ordinary physical space and the 3-dimensional forces $\left\{-\vec{\nabla}_{q_{k}}(\mathrm{U}(q, t)+\mathrm{V}(q, t))\right\}$ that appear on the right-hand side of MNSL. As the existence of the quantum field is explicitly denied, these forces are deemed irreducibly primitive. ${ }^{22}$

It is important to note that Belousek interprets the forces $\left\{-\vec{\nabla}_{q_{k}}(\mathrm{U}(q, t)+\mathrm{V}(q, t))\right\}$ not as properties of the particles, but as entities that are distinct from them. Hence, he takes himself to be embracing a dualistic ontology of particles and 3-dimensional forces that act upon the particles, accelerating them as prescribed by MNSL. Similarly to what happens in Newtonian mechanics, Belousek considers that the motion of Bohmian particles in a given situation is explained by the specific arrangement of forces present in that situation. If, for instance, we again consider the double slit experiment, it is the presence of a primitive quantum force that causes the twists and bounces in the trajectory of the test particle. In light of this causal story, Belousek concludes that his interpretation meets desideratum (iii).

There are certain similarities between the ontological framework posited by Belousek and that of Newtonian mechanics. Since the quantum force $-\vec{\nabla}_{q_{k}} \mathrm{U}(q, t)$ is a function of the quantum state and the latter depends on the positions of every particle, it follows that the value of the force at an instant, $t$, depends on the value of the positions of all the particles at $t$. Hence, the forces $-\vec{\nabla}_{q_{k}} \mathrm{U}(q, t)$ act at a distance exactly like Newton's gravitational forces. In addition, quantum forces are deemed primitive as happened with Newton's gravitational forces. Belousek readily recognizes these similarities:

One would have, then, a genuine dualistic ontology -equi-primordial particles and forces. Of course, one is left here without an account of the origin of such forces;

\footnotetext{
${ }^{22}$ It is worth noting that Belousek's idea is not completely original. As early as 1954-just a couple of years after the publication of the seminal papers on Bohmian mechanics-Margenau commented on the new interpretation in terms of hidden variables championed by Bohm. In a list of alleged difficulties with the theory, Margenau declared that: "the state function cannot represent physical reality because it is complex, and it extends in configuration space of many dimensions, not in ordinary space," but he readily dismissed the objection with the following caveat: "this argument can be met by supposing that the forces conveyed by the non-classical field are complicated many-body forces" (Margenau 1954, p. 8). For other reasons, Margenau did not finally favor Bohmian mechanics but committed himself to a 'latency interpretation' of quantum mechanics. For more on Margenau's interpretation, see Suárez (2007, Section 2).
} 
and, for sure, the notion of 'primitive force' remains as mysterious now as it was to Newton and his contemporaries. (Belousek 2003, p. 163)

The parallels between classical forces and the quantum forces appearing on the right-hand side of MNSL end here; and there are indeed important differences between the two sorts of forces. First, as I point out in $\S 3$ above, the quantum forces arising from the quantum potential are generally many-body rather than two-body forces and do not need to fall off with distance. There is, however, a far more important dissimilarity. Despite the fact that Newton did not make hypotheses about the origin of gravity, Newtonian gravitational forces are pre-assigned functions of inter-particular distances and masses, so there can be no ambiguity in considering that the source of the forces are the particles themselves and, more specifically, their masses. This is not true of the quantum forces $-\vec{\nabla}_{q_{k}}(\mathrm{U}(q, t))$. Since they depend on the modulus of the wave function, these forces do not only supervene on the positions of particles but they covary with the quantum state. Since Belousek has expunged the wave function from the ontology, he must accept that the quantum forces have no source.

Belousek is perfectly aware of positing unsourced quantum forces and it is because of the counterintuitive character of this that he takes his proposal to be somehow "provisional" and "awaiting a better physical interpretation of the quantum potential in accord with our desiderata" (2003, p. 163). Interestingly, I think that it is precisely DGZ's attempt to interpret the wave function as nomological in nature what could keep Belousek's interpretation out of harm. Notice that if the wave function were-as DGZ would like it to be- a quantum-mechanical parameter with a role analogous to that of the classical Hamiltonian, then the quantum potential would have exactly the characteristics that we expect of a potential function: it would be a static, noncontingent function, unambiguously determined (up to a constant) given the contents of the universe. In such a case, the quantum forces arising from the quantum potential could be safely interpreted as many-body forces; certainly complicated, but having their source in the particles themselves. Hence, although Belousek introduces his proposal in a clear dialectic with the rival three-dimensionalist interpretation of DGZ, both interpretations seem ultimately to share the same fate; since the easier it is to interpret 
the wave function as a law, the more plausible Belousek's postulation of quantum forces without the wave field becomes. ${ }^{23}$

\section{Suárez's propensity interpretation of Bohmian mechanics}

Suárez (2007) proposes a 3-dimensionalist interpretation of Bohmian mechanics that is different from Belousek's but similarly operates within the quantum potential approach. Suárez's interpretation is closely connected to his defense of a selective propensities interpretation of ordinary non-relativistic quantum mechanics (see Suárez $2004 a$ and 2004b). I will briefly recap the main ideas of Suárez's propensity interpretation of standard quantum theory before considering his proposal concerning Bohmian mechanics.

Suárez attributes a full catalogue of 'selective propensities' to every quantum system. A propensity is an objective dispositional property of a system that entails a manifestation with a specific probability if certain conditions apply. ${ }^{24}$ Therefore, making a commitment to propensities essentially amounts to accepting brute objective chances in the ontology, no matter how they are to be interpreted. Selective propensities, in particular, are specifically related to measurements, since they manifest themselves through an actual value of a physical property being obtained when that property is measured. Suárez claims that propensities have a very fundamental explanatory role, since it is the actual possession of a set of selective propensities on the part of a system that explains the outcomes of the measurements performed on that system.

\footnotetext{
${ }^{23}$ This situation invites one to think of a nomological interpretation that would be an alternative to that of DGZ. The basic idea underlying such an interpretation would be to take the MNSL as the fundamental law of motion and to interpret, not the wave function, but the quantum potential (or the modulus of the wave function, $\mathrm{R}$ ) as a parameter in the law. This alternative interpretation may turn out to have certain advantages over that of DGZ. It is worth noting, in this respect, that there are time-dependent wave functions that give rise to a static quantum potential. This happens, for instance, when the wave function is stationary. Consequently, interpreting the quantum potential as a parameter in the law may be easier to motivate than interpreting the wave function in this way.

${ }^{24}$ More concretely, Suárez $(2007$, p. 430) analyzes propensities by means of the following conditional entailment: "If object $\mathrm{O}$ possesses propensity $\mathrm{P}$ with manifestation $\mathrm{M}$, were $\mathrm{O}$ to be tested (under the appropriate circumstances $\mathrm{C} 1 ; \mathrm{C} 2 ; \ldots)$ it would $\mathrm{M}$ with probability $p, 0 \leq p \leq 1$ ". Notice that the particular case in which the manifestation occurs with certainty $(p=1)$ is included in the conditional. Therefore, Suárez considers sure-fire dispositions (referred to as 'deterministic propensities' by him) to be a limiting case of the more general notion of 'disposition'.
} 
It is crucial to Suárez's project that an adequate formal representation of each quantum propensity be found. Since the quantum state, $\Psi$, of a system entails a probability distribution for each observable, $\mathrm{O}$, defined in the Hilbert space of the system, then $\Psi$ includes information about all the selective propensities of the system. Therefore, $\Psi$ is not a good candidate to represent a specific selective propensity. Suárez's final candidate for such formal representation is that, if we consider a system in state $\Psi$ and a (discrete, not maximally degenerate) observable $\mathrm{O}$ of that system with spectral decomposition such that $\mathrm{O}=\sum_{\mathrm{n}} o_{n} \mathrm{P}_{\left|o_{n}\right\rangle} \equiv \sum_{\mathrm{n}} o_{n}\left|o_{n}\right\rangle\left\langle o_{n}\right|$, then the propensity of the system associated with $\mathrm{O}$ is adequately represented by the so-called standard representative, $\mathrm{W}_{\Psi}(\mathrm{O})$, given by:

$$
\mathrm{W}_{\Psi}(\mathrm{O})=\sum_{n} \operatorname{Tr}\left(\Psi\left|o_{n}\right\rangle\left\langle o_{n}\right|\right) \frac{\left|o_{n}\right\rangle\left\langle o_{n}\right|}{\operatorname{Tr}\left(\left|o_{n}\right\rangle\left\langle o_{n}\right|\right)}
$$

The minimal motivation for such a claim is that $\mathrm{W}_{\Psi}(\mathrm{O})$ entails the very same statistics as regards the observable $\mathrm{O}$ as $\Psi$ itself does, but clearly it is not as informative as $\Psi$ is with respect to observables that are not functions of $\mathrm{O}$.

Suárez contends that one of the main advantages of introducing selective propensities is that they amount to a solution of the measurement problem. The main gist of his idea is as follows. ${ }^{25}$ Quantum measurements are selective in the sense that it is one property of the object system, not all of them, that is being discriminated. In this regard, it seems plausible to consider the measurement to be an interaction between the position of the pointer in the apparatus and the specific property of the system that is under test. Notice, however, that according to standard quantum measurement theory, the measurement interaction is represented as:

$$
\Psi \otimes \Phi_{a} \rightarrow \mathrm{U}_{t}\left(\Psi \otimes \Phi_{a}\right) \mathrm{U}_{t}^{-1}
$$

that is, the unitary Schrödinger time evolution of the composite formed by the initial state of the object, $\Psi$, and the initial state of the apparatus, $\Phi_{a}$, assuming a suitable Hamiltonian. Since the quantum state, $\Psi$, encodes the probability distributions of all the observables of the object system, Suárez considers that the above expression suggests the apparatus interacts with all propensities of the object system and that, in consequence, it misrepresents the alleged selective character of the measurement. A

\footnotetext{
${ }^{25}$ Here, Suárez basically draws on Fine $(1987 ; 1992)$.
} 
selective measurement, he claims, would be rightly represented if the initial state was fed in with the standard representative that characterizes the propensity of the system being tested. In such a case, the measurement interaction would be modeled as:

$$
\mathrm{W}_{\Psi}(\mathrm{O}) \otimes \Phi_{a} \rightarrow \mathrm{U}^{*}\left(\mathrm{~W}_{\Psi}(\mathrm{O}) \otimes \Phi_{a}\right) \mathrm{U}^{*-1}
$$

where the operator $U^{*}$ represents the unitary Schrödinger time evolution that is suitably generalized in order to apply to mixtures, and $\mathrm{O}$ is the observable to be measured. As $W_{\Psi}(O)$ is either an eigenstate of $\mathrm{O}$ or a mixture of different eigenstates of $\mathrm{O}$, the final post-measurement wave function cannot be an entangled superposition of different eigenstates of the position of the pointer in the apparatus and therefore no difficulties arise in attributing a definite pointer reading to such a state. To sum up, Suárez's solution to the measurement problem consists of invoking the alleged selective character of quantum measurements to motivate a modification of standard quantum measurement theory that amounts to a suitable restriction on the set of initial premeasurement states that are allowed.

In his 2007, Suárez attempts to show that selective propensities or similar dispositional notions can usefully be applied to other interpretations of quantum mechanics. More specifically, he discusses the implementation of such notions in the collapse theory of Ghirardi et al. (1986) and Bohmian mechanics, offering a small preview of a more ambitious project yet to be developed that would demonstrate the consistency of dispositions with all interpretations of quantum theory.

The introduction of propensities in a theory like Bohmian mechanics prima facie seems suspicious. First, since Bohmian mechanics is a deterministic theory, the propensities of a Bohmian particle need to be sure-fire dispositions. This is not a problem for Suárez, who considers sure-fire dispositions to be a subspecies of propensities; but the appeal and the richness of the notion seems to be clearly diminished if we restrict propensities to the deterministic case. Moreover, since in Bohmian mechanics there is no measurement problem, a propensity interpretation of the theory cannot be motivated by its role in solving the measurement problem. In light of this, one wonders what the reason for introducing propensities into Bohmian mechanics could be to begin with. As I aim to show below, it turns out that one of Suárez's main motivations, as it was for Belousek, is to provide an interpretation of the theory that is both 3-dimensionalist and adequate from the point of view of explanation. 
Before introducing his own proposal, Suárez undertakes a brief critical review of some interpretations of Bohmian mechanics. DGZ's is the first view he discusses and he characterizes it as an interpretation that takes the Bohmian trajectories given by GE as the phenomena "and refrains from making any additional suppositions regarding the causal or explanatory structure that might underpin and give rise to such phenomena" (2007, p. 435). Suárez does not find the sparse ontological framework envisaged by DGZ a homely environment for entities such as his propensities, whose main role is explanatory, and goes on to discuss the quantum potential approach to the theory. Thus, he comments on Holland (1993), an author who is explicitly committed to the existence of the quantum potential and the associated force field in configuration space. Although Suárez finds this interpretation to be more explanatory than that of DGZ, he dismisses it because of the untenability of configuration space realism. Instead of interpreting the force field defined by MNSL as a separate physical entity in configuration space, Suárez proposes to interpret the modalities described by such a field as propensities of the Bohmian particles:

My suggestion would be to reinterpret the quantum potential and force field along similar lines except the modalities described by the quantum wave function in configuration space now describe a full catalogue of the propensities of the system. (Suárez 2007, p. 436)

This quotation also makes it clear that Suárez embeds his propensity interpretation of Bohmian mechanics within the quantum potential approach to the theory. There are other noteworthy features of Suárez's Bohmian propensities. First, he considers that Bohmian propensities are selective propensities that can be represented using the standard representative (25). Therefore, there is no difference between how propensities are formally dealt with in the context of Bohmian mechanics and in that of quantum mechanics. ${ }^{26}$ Second, the highly non-local character of Bohmian mechanics readily translates into a non-local instantiation of Bohmian propensities. Third, the very trajectories of the Bohmian particles are considered to be the result of their propensities:

\footnotetext{
${ }^{26}$ For instance, when commenting on a two-particle Bohmian system, Suárez (2007, p. 436) claims: "In the case of the two particle system formed by a quantum object subject to an interaction with a measuring device, this boils down to writing down each and every possible interaction between the measurement device and the propensities $\left\{\mathrm{O}_{1}, \mathrm{O}_{2}, \ldots, \mathrm{O}_{\mathrm{n}}\right\}$ of the quantum system described by its corresponding standard representatives $\left\{\mathrm{W}\left(\mathrm{O}_{1}\right), \mathrm{W}\left(\mathrm{O}_{2}\right), \ldots, \mathrm{W}\left(\mathrm{O}_{\mathrm{n}}\right)\right\}$."
} 
Interesting complications will arise in the case of $n$-particle systems subject to measurements. In these cases the trajectories of each of the particles (the only observable consequences of the theory according to Bohm) would be the result of not just the selective propensities of each particle, but also the selective propensities of all the other particles as described through the quantum potential and the resulting force field would be the result of all the selective-propensities and their mutual interactions. (Suárez 2007, p. 436)

Summing up, Suárez explicitly rejects reifying the wave function or any other field in configuration space. Instead he endows Bohmian particles with a full set of deterministic selective propensities, interpreted as primitive dispositional properties that entail which outcomes would obtain, were the particles to interact with measuring devices. These propensities are credited with a fundamental explanatory role, since the trajectories of the particles are ultimately accounted for in terms of their propensities and their interaction with the propensities of other particles. Given that no fields are postulated in configuration space, Suárez's interpretation meets the 3-dimensionalist requirement. Now, since the wave function determines the objective propensities of the particles which, in turn, account for the particles' trajectories, it seems safe to conclude that this interpretation satisfies Belousek's third desideratum. In this regard, the introduction of propensities in Bohmian mechanics responds to the very same motivation that led Belousek to introduce unsourced primitive forces and it can be agreed that, in the context of devising a 3-dimensionalist interpretation of the theory, Suárez's interpretive maneuver has a certain explanatory appeal.

Still, many aspects of Suárez's proposal are in need of further clarification. For instance, Suárez does not make it clear why the sure-fire dispositions of Bohmian particles should be interpreted as selective propensities; and in fact, such a requirement may turn out to introduce unnecessary complications. As Suárez characterizes them, selective propensities are strongly linked to measurements because they manifest themselves precisely when measurements occur. Conceptually, the stress on measurements somehow seems to contradict the spirit of Bohmian mechanics; which counts among its most notable virtues (if contrasted with the standard quantum mechanical approach) the fact that measurements can be seen as a subspecies of 
physical interactions with no distinguished ontological status. ${ }^{27}$ It should also be noted that in Bohmian mechanics properties are represented as sets of points in configuration space, whereas in standard quantum theory they are represented as subspaces of Hilbert space. Therefore, prima facie it does not seem plausible that a Bohmian propensity could be properly represented by the standard representative (25); a formal characterization the rationale for which is entirely dependent on the altogether different way properties are represented in the standard approach. Finally, the very idea of a selective interaction, which motivates the introduction of selective propensities in the standard approach, does not fit in well with Bohmian mechanics. According to Suárez, a selective interaction is an interaction that takes place with only one property of the object: not with the others. Since, other than positions, the properties of Bohmian particles supervene on both the positions themselves and on the wave function, and moreover, the latter is irreducibly modified in the course of a measurement, it follows that in the course of a Bohmian measurement all the properties of the object are modified-including the one being measured. In light of this, Bohmian measurements are not selective but constitute interactions with all the properties of the object. ${ }^{28}$ These difficulties do not obviously show that postulating propensities is inconsistent with Bohmian mechanics, but it may turn out that postulating selective propensities is.

\section{Two interpretive alternatives presented}

The DGZ interpretation is the only three-dimensionalist interpretation of Bohmian mechanics within the guidance approach that has appeared in the literature to date and, as we have just seen, it has been dismissed as explanatorily wanting by Belousek and Suárez. In order to offer a three-dimensionalist interpretation of the theory that is more satisfactory from the point of view of explanation, both Belousek and Suárez draw on resources that are characteristic of the quantum potential approach. Thus, Belousek contends that his own interpretive proposal is more explanatory than the DGZ interpretation due to the postulate of primitive forces that appear on the right-hand side of MNSL and to its having available the dynamic concepts and explanatory resources

\footnotetext{
${ }^{27}$ See, for instance, Bohm and Hiley (1993: Chapter 6).

${ }^{28}$ Perhaps the completion of Suárez's project would require a modification of Bohmian measurement theory, but he does not mention such a possibility.
} 
characteristic of classical mechanics. Similarly, Suárez considers that attributing a full catalogue of selective propensities to Bohmian particles renders his interpretation superior in explanatory power to that of DGZ, and he explicitly links the propensities with the modalities conveyed by MNSL and the associated force field in configuration space. If we agree that Belousek's and Suárez's proposals fare better than DGZ's interpretation in explanatory terms, we may be tempted to conclude that the quantum potential approach has better prospects than the guidance view when it comes to articulating an interpretation of Bohmian mechanics that is both three-dimensionalist and satisfactory from the standpoint of explanation. This is actually Belousek's own verdict; while acknowledging some of the weaknesses of his own proposal, he nevertheless claims:

None of the above views are without some difficulty with respect to our desiderata for the interpretation of Bohmian mechanics [...] However, the arguments presented above concerning explanatory adequacy and a coherent classical limit do mitigate against DGZ guidance view and point in favor of an interpretation within the causal view. (i.e., an interpretation that accords secondorder dynamics and the quantum potential physical significance in some sense.) (Belousek 2003, pp. 164-165)

Suárez similarly concludes:

Thus the propensity interpretation of Bohmian mechanics has all the advantages associated to the "causal" views of Bohmian mechanics, in particular its superior explanatory power in comparison with "guidance" views; but it purchases these advantages at a lesser ontological cost. (Suárez 2007, p. 436)

My aim in this paper is to show-contrary to what the two authors just quoted suggest - that the quantum potential approach does not have more explanatory power than the guidance approach; and that the desiderata of a tenable three-dimensionalist ontology and adequate explanation do not provide grounds for favoring the quantum potential approach. I have already argued, in this respect, that some of the criticisms raised against DGZ are unfair in that they overlook the attempt made by DGZ at a nomological interpretation of the wave function and those criticisms further dismiss the possibility of nomologically-based explanations of Bohmian phenomena. In what 
follows, I aim to show that even if one accepts the accounts of explanation defended by Belousek and Suárez, the quantum potential approach need not be favored. I do this by advancing two three-dimensionalist interpretations of Bohmian mechanics that draw only on resources that are available to supporters of the guidance view and that are at least as explanatory as Belousek's and Suárez's proposals according to their own standards of explanation. As we will see immediately, the key to devising such interpretations is to rely not on the ontological framework provided by DGZ, but on the dynamical reinterpretation of the guidance approach in terms of Aristotelian forces developed by Valentini (1997) and which I explore in some detail in $\S 3$ above.

Valentini is a configuration space realist who takes the function $\mathrm{S}$ - the phase of the wave function or the pilot wave - to represent a real (but subtle) physical field which informs the movement of Bohmian particles. He assumes that GE is the fundamental law of motion of Bohmian particles and interprets the action of the pilot wave upon the particles as mediated by Aristotelian forces that are proportional to the velocities of the particles. The causal-dynamical structure of such an interpretation has three levels, since we have a quantum field (in configuration space) that is the source of threedimensional forces which, in turn, account for the trajectories of the particles. In this respect, Valentini's fully-fledged realistic interpretation within the guidance approach is completely analogous to other fully-fledged realistic interpretations of the theory that operate within the quantum potential approach, such as those of Bohm and Hiley (1993) and Holland (1993).

Belousek (2003) takes as his starting point a fully-fledged realistic interpretation of the theory within the quantum potential approach but, instead of reifying the wave field, he postulates the existence of three-dimensional forces $\left\{-\vec{\nabla}_{q_{k}}(\mathrm{U}(q, t)+\mathrm{V}(q, t))\right\}$ that appear on the right-hand side of MNSL, reinterpreted as primitive dynamical agents. Now, this strategy can be mimicked perfectly in the context of Valentini's fully-fledged realistic interpretation within the guidance approach. The result is a dualistic interpretation of Bohmian mechanics committed to the existence of both the Bohmian particles in three-dimensional space and, over and above the particles, the set of threedimensional Aristotelian forces $\left\{\vec{\nabla}_{q_{k}} \mathrm{~S}(q, t)\right\}$ that appear on the right-hand side of GE. In what follows, I will refer to such an interpretation as [INT1]. Here, as in Belousek's own interpretation, the existence of the wave field is explicitly denied and, consequently, the Aristotelian forces $\left\{\vec{\nabla}_{q_{k}} \mathrm{~S}(q, t)\right\}$ are deemed primitive. Since [INT1] is 
committed to the existence of Aristotelian forces, Valentini's arguments showing the consistency of such a postulate with the alleged Galilean invariance of Bohmian mechanics also apply to this interpretation. Therefore, according to [INT1], Galilean invariance is not a fundamental symmetry of the theory. Despite the evident similarities, this interpretive proposal is not the same as that of Valentini himself, since Valentini is a configuration space realist.

The causal-dynamical structure of [INT1] is completely analogous to that of Belousek's interpretation. According to [INT1], the primitive Aristotelian force $\vec{\nabla}_{q_{k}} \mathrm{~S}(q, t)$ acts upon the $k$ th particle causing it to deviate from absolute rest, whereas in Belousek's interpretation, it is the primitive Newtonian force that acts upon the $k$ th particle accelerating it. Hence, in both interpretations we have primitive forces (Aristotelian or Newtonian) that account for the motion of the particles and, in both, the forces (Aristotelian or Newtonian) formally depend on the wave function, which is declared inexistent but can be attributed an indirect physical meaning through this dependence. Consequently, anyone who accepts that Belousek's interpretation meets Belousek's own explanatory desideratum (iii), must also accept that this desideratum is met by [INT1]. Unfortunately, [INT1] does not only share the explanatory strengths of Belousek's proposal but it also shares some of its weaknesses and, exactly as happens with the primitive Newtonian forces in Belousek's account, the primitive Aristotelian forces postulated in [INT1] cannot be taken to have the particles as a source. Therefore, all the difficulties associated with the postulate of unsourced forces (and their possible resolutions) also afflict my alternative proposal.

Next, I consider Suárez's (2007) propensity interpretation of Bohmian mechanics. I show in $\S 6$ above that this interpretation is explicitly grounded in the quantum potential approach to the theory. I think one of the reasons that led Suárez to such a position is that he conflates the guidance view with the ontologically sparse interpretation of DGZ. Yet nothing in the possibility of attributing dispositions to Bohmian particles seems to depend on whether the dynamics of the particles is first-order or second-order. In this respect, if, as Suárez explicitly contends, the modalities described by the (Newtonian) force field defined by the quantum potential can be interpreted as a catalogue of propensities of Bohmian particles, then so can the modalities described by the (Aristotelian) force field $\nabla_{q} \mathrm{~S}(q, t)$. This latter interpretation would lead to a propensity 
interpretation of Bohmian mechanics that is fully embedded within the guidance approach. In what follows, I will refer to this interpretation as [INT2].

The reader may wonder to what extent Suárez's interpretation and [INT2] differ. I maintain that they do, since the modalities conveyed by MNSL and those conveyed by GE are not the same. As I show in $\S 3$ above, MNSL admits many more solutions than GE does and, therefore, it sanctions as possible certain trajectories of Bohmian particles that are forbidden by GE. Now, given that Suárez postulates a catalogue of propensities that corresponds with the modal space conveyed by MNSL but [INT2] is only committed to those propensities that correspond to the more reduced set of modalities conveyed by GE, it follows that the interpretations differ and that [INT2] is sparser in its ontological claims.

Suárez's interpretive proposal is of interest due to the alleged explanatory task carried out by the selective propensities which are taken ultimately to account for the trajectories of the particles and therefore for the outcomes of measurements performed upon the particles. There is, however, no fundamental explanatory difference between the propensities posited by Suárez and those posited in [INT2]. Hence, anybody who agrees with Suárez that his interpretation is adequate from the standpoint of explanation must concede that [INT2] is too. In fact, given that the ultimate explanandum is the same for both interpretations, a parsimony argument may be used to favor [INT2], since it explains the same as Suárez does but uses fewer resources.

For similar reasons, it can be argued that [INT1] has certain explanatory advantages over Belousek's interpretation. The core of the issue resides, once again, in the fact that MNSL has surplus content compared to GE and that only those solutions of the former that are also solutions of the latter are compatible with the statistical postulate (BM4). Hence, defenders of the quantum potential approach still need to resort to GE and interpret it as constraint (8) on the initial conditions. Now, given that the only dynamical agents in Belousek's ontology are the three-dimensional forces $\left\{-\vec{\nabla}_{q_{k}}(\mathrm{U}(q, t)+\mathrm{V}(q, t))\right\}$ that appear on the right-hand side of MNSL, the fact that the actual Bohmian trajectories all satisfy constraint (8) seems really conspiratorial and calls for an explanation that Belousek's interpretation does not provide. In contrast, if it is the set of three-dimensional Aristotelian forces that appear on the right-hand side of GE and exist over and above the particles, as [INT1] requires, then constraint (8) 
matches the dynamical possibilities allowed by the ontology perfectly and no explanation is called for.

This objection to Belousek's interpretation can be illustrated by considering the case of atomic s-states; a case that Belousek himself discusses. ${ }^{29}$ In an $s$-state, the quantum force arising from the quantum potential and the Coulomb force are perfectly balanced. Hence, there is no net force acting on the electron and, therefore, the electron cannot be accelerated. An unaccelerated electron does not radiate and, consequently, we have a very nice explanation of why atoms are stable. In addition, it turns out that the ground state is the lowest energy state in which such a balance of forces occurs, since for energies below that of the ground state, the repulsive effect of the quantum force would exceed the Coulomb attraction. This explains, in addition, why there is no stationary energy state below the ground state.

Beautiful as this explanatory story is, it cannot be the whole story. If there is no net force, all the trajectories of the electron with constant finite velocity (relative to the nucleus) are possible according to MNSL. However, all those trajectories would imply that the electron drifts away from the nucleus which is contradictory to the facts and the very idea of atomic stability. The only trajectory that ensures stability is the one that takes the electron to be at rest and, in fact, since in all $s$-states the phase, $\mathrm{S}$, of the wave function is constant, it is a straightforward consequence of GE that in those states the electron is always at rest.

The only 'explanation' that Belousek can offer regarding this notorious fact consists of invoking a gigantic conspiracy of initial conditions. This maneuver, however, merely amounts to acknowledging the fact, but not to explaining it. Now, the supporter of [INT1] can explain why all electrons in $s$-states are at rest perfectly. Since in those states the phase, $S$, of the wave function is constant, its gradient is zero and therefore there is no net Aristotelian force acting on the electron. If that is so, it simply follows that the electron must be at rest.

\section{Conclusions}

DGZ defend the idea that the wave function is a component of the law of motion of particles and account for Bohmian trajectories merely in terms of that law, which they

\footnotetext{
${ }^{29}$ See Belousek (2003, pp. 139ff).
} 
take to be underwritten by GE. Belousek and Suárez prefer to enrich the ontology so they can provide a less abstract account of the trajectories. Belousek considers that the trajectories are adequately explained in terms of the action of the three-dimensional Newtonian forces appearing on the right-hand side of MNSL, interpreted as primitive causal agents. Similarly, Suárez takes the Bohmian trajectories to be the result of the propensities of the particles and links those propensities with the modalities defined by MNSL.

The explanatory strategies of Belousek and Suárez can be mimicked perfectly by defenders of the guidance approach, provided that they rely on the dynamic reinterpretation of GE in terms of Aristotelian forces developed by Valentini. That leads to the three-dimensionalist interpretations [INT1] and [INT2], according to which the Bohmian trajectories are accounted for either by the three-dimensional Aristotelian forces appearing on the right-hand side of GE or by the propensities of the Bohmian particles corresponding to the modalities allowed by that very same equation. Not only do [INT1] and [INT2] mirror the causal and explanatory structure advocated by Belousek and Suárez respectively, thus warranting them at least the same explanatory power, but I have also shown that postulating Aristotelian forces in Bohmian mechanics accrues some explanatory advantages. Hence it can safely be concluded that the desiderata of a three-dimensionalist ontology and adequate explanation does not favor the quantum potential approach to Bohmian mechanics.

None of the interpretations surveyed here is, however, without problems. In motivating the nomological interpretation of the wave function, DGZ go far beyond the limits of non-relativistic Bohmian mechanics and anyone wanting to take their interpretation seriously while being fair to the postulates of the theory, must be ready to revise the classical notion of law. Unsourced quantum forces are posited both in Belousek's interpretation and in [INT1]; whereas postulating Bohmian propensities could seem rather ad hoc to many. Yet this only means that more interpretive work is needed and it may turn out that one is quite willing to pay either price in order to interpret Bohmian mechanics without wave function ontology. 


\section{Acknowledgements}

I would like to thank Carl Hoefer, Xavier Oriols and Laura Felline for helpful comments on previous drafts of this paper. I am also grateful to the audiences of the 2011 Annual Meeting of the British Society for the Philosophy of Science, the $14^{\text {th }}$ Congress of Logic, Methodology and Philosophy of Science and the VII Conference of the Society of Logic, Methodology and Philosophy of Science in Spain for many useful suggestions. Research towards this paper has been funded by the Spanish Ministry of Science and Innovation Consolider-Ingenio 2010 Scheme CSD2009-00056 and the research project FFI2011-29834-C03-03.

\section{References}

Albert, D. Z. (1996). Elementary Quantum Metaphysics. In Cushing, J. T., Fine, A., \&

Goldstein, S. (Eds.), Bohmian mechanics and quantum theory: An appraisal (pp. 277284). Dordrecht: Kluwer Academic Press.

Baublitz, M., \& Shimony, A. (1996). Tension in Bohm's Interpretation of Quantum Mechanics. Cushing, J. T., Fine, A., \& Goldstein, S. (Eds.), Bohmian mechanics and quantum theory: An appraisal (pp. 251-264).

Bell, J.S. (1987). Speakable and Unspeakable in Quantum Mechanics. Cambridge: Cambridge University Press.

Bell, J.S. (1990). Against Measurement. Physics World, 3, 33-40.

Belot, G. (2012). Quantum States for Primitive Ontologists. European Journal for Philosophy of Science, 2(1), 67-83.

Belousek, D.W. (2003). Formalism, Ontology and Methodology in Bohmian Mechanics. Foundations of Science, 8(2), 109-172.

Bohm, D., (1952). A Suggested Interpretation of the Quantum Theory in Terms of ‘Hidden' Variables I and II. Physical Review, 85, 166-193. 
Bohm, D., \& Hiley, B.J. (1993). The Undivided Universe: An Ontological Interpretation of Quantum Theory. London: Routledge \& Kegan Paul.

Brown, H.R., Elby, A., \& Weingard, R. (1996). Cause and Effect in the Pilot-wave Interpretation of Quantum Mechanics. In Cushing, J. T., Fine, A., \& Goldstein, S. (Eds.), Bohmian mechanics and quantum theory: An appraisal (pp. 309-319).

Dordrecht: Kluwer Academic Press.

De Witt, B.S. (1967). Quantum Theory of Gravity. I. The Canonical Theory. Physical Review, 160 (5), 1113-1148.

Dürr, D., Goldstein, S., \& Zanghì, N. (1992). Quantum Equilibrium and the Origin of Absolute Uncertainty. Journal of Statistical Physics, 67, 843-907.

Dürr, D., Goldstein, S., \& Zanghì, N. (1997). Bohmian Mechanics and the Meaning of the Wave Function. In Cohen, R. S., Horne, M. \& Stachel, J. (Eds.), Experimental Metaphysics - Quantum Mechanical Studies for Abner Shimony, Volume One; Boston Studies in the Philosophy of Science 193 (pp. 25-38). Boston: Kluwer Academic Publishers.

Fine, A. (1987). With complacency or concern: Solving the quantum measurement problem. In Kargon, R. \& Achistein, P. (Eds), Kelvin's Baltimore lectures and modern theoretical physics: Historical and philosophical perspectives (pp. 491-505).

Cambridge, MA: MIT Press.

Fine, A. (1992). Resolving the Measurement Problem: A Reply to Stairs. Foundations of Physics Letters, 5, 125-38.

Ghirardi, G., Rimini, A., \& Weber, T. (1986). A model for a unified quantum description of macroscopic and microscopic systems. Physical Review D, 34, 470.

Goldstein, S., \& Zanghì, N. (2013). Reality and the Role of the Wave Function in Quantum Theory. In Ney, A. \& Albert D.Z. (Eds), The Wave Function: Essays on the Metaphysics of Quantum Mechanics (pp. 91-109). New York: Oxford University Press. 
Holland, P.R. (1993). The Quantum Theory of Motion: An Account of the De BroglieBohm Causal Interpretation of Quantum Mechanics. Cambridge: Cambridge University Press.

Jammer, M. (1999). Concepts of Force: a Study in the Foundations of Dynamics.

Mineola, New York: Dover Publications.

Lewis, P.J. (2004). Life in Configuration Space. British Journal of Philosophy of Science, 55, 713-729.

Margenau, H. (1954). Advantages and disadvantages of various interpretations of the quantum theory. Physics Today, 7(10), 6-13.

Maudlin, T. (2010). Can the World be Only Wavefunction?. In Saunders, S., Barrett, J., Kent, A., \& Wallace, D. (Eds.) Many Worlds? Everett, Quantum Theory, \& Reality (pp. 121-43). Oxford: Oxford University Press.

Monton, B. (2002). Wave Function Ontology. Synthese, 130, 265-277.

Monton, B. (2006). Quantum Mechanics and 3N-Dimensional Space. Philosophy of Science, 73, 778-789.

Suárez, M. (2004a). On quantum propensities: two arguments revisited. Erkenntnis, 61, $1-16$.

Suárez, M. (2004b). Quantum selections, propensities, and the problem of measurement. British Journal for the Philosophy of Science, 55, 219-255.

Suárez, M. (2007). Quantum Propensities. Studies in the History and Philosophy of Modern Physics, 38, 418-438.

Valentini, A. (1992). On the Pilot-Wave Theory of Classical, Quantum and Subquantum Physics. Ph.D. Dissertation, ISAS - International School for Advanced Studies, Trieste.

Valentini, A. (1997). On Galilean and Lorentz Invariance in Pilot-Wave dynamics. Physics Letters, A228, 215-222. 